Original Article

\title{
COMPUTATIONAL DOCKING AND IN SILICO ANALYSIS OF POTENTIAL EFFLUX PUMP INHIBITOR PUNIGRATANE
}

\author{
ZUMAANA RAFIQ ${ }^{1}$, SARANYA SIVARAJ ${ }^{1}$, RAMA VAIDYANATHAN ${ }^{2 *}$
}

${ }^{1}$ Department of Biotechnology, Dr. M. G. R. Educational and Research Institute, E. V. R. Periyar Salai, Maduravoyal, Chennai 600095, ${ }^{2}$ Dr. A. P. J. Abdul Kalam Centre for Innovation and Entrepreneurship, Dr. M. G. R. Educational and Research Institute, I Year Campus, E. V. R. Periyar Salai, Adayalampattu, Chennai 600095

Email: ramavaidy@gmail.com

Received: 26 Sep 2017 Revised and Accepted: 11 Jan 2018

\begin{abstract}
Objective: Efflux-mediated resistance is a growing therapeutic complication as it reduces the efficacy of antibiotics. In gram-negative bacteria like $E$. coli and K. pneumoniae, this can be overcome with the help of efflux pump inhibitors (EPI) targeted at the transporter protein AcrB that plays a key role in binding to antibiotics. Our study focuses on the potential EPI Punigratane isolated from the rind of Punica granatum. Using computational docking analysis and in silico analysis, our aim is to determine whether Punigratane has the ability to interact and inhibit the AcrB pump and whether it has drug viability.
\end{abstract}

Materials: Computational docking analyses were carried out using the online platforms Mcule and PatchDock. Drug-likeness and classification of Punigratane was predicted using online tools PreADMET and SuperPred. Admet SAR and Toxicity Checker at Mcule were used to predict ADME (absorption, distribution, metabolism, and excretion) and overt toxicity properties.

Results: Punigratane was computationally docked with 57 AcrB crystal structures available at the PDB database to determine whether it could bind to the active site regions. It was found to bind in the periplasmic region close to the substrate bile acid where it is thought to bring about inhibition by steric hindrances. When docked with AcrB mutant (AcrB N109A), it was found to bind in the same periplasmic site as the substrates (EtBr, Rhodamine 6G, Ciprofloxacin, Bile acid) as well as the inhibitor (phenylalanine-arginine $\beta$-naphthylamide-Pa $\beta N$ ). When docked in the active site of the inhibitor MBX2319, it was found to have a comparable docking score as well as the same hydrophobic interactions as the inhibitor. In silico analysis showed that Punigratane exhibited a drug-likeness to the inhibitor MBX2319 and that its drug classification is similar to antimicrobial agents. It was also found be a potential drug due to its intestinal absorption, increased bioavailability and non-toxic nature.

Conclusion: Therefore our report shows that Punigratane could be a potential drug candidate that inhibits efflux activity by interacting and inhibiting the AcrB efflux pump.

Keywords: Punigratane, EPI, AcrB efflux pump, Drug candidate, Docking

(C) 2018 The Authors. Published by Innovare Academic Sciences Pvt Ltd. This is an open access article under the CC BY license (http://creativecommons.org/licenses/by/4.0/] DOI: http://dx.doi.org/10.22159/ijpps.2018v10i3.21629

\section{INTRODUCTION}

Antibiotic resistance, the silent pandemic, is taking the world by storm. Among the various factors that contribute to the resistant nature of bacteria, resistance via efflux pumps is a growing therapeutic complication. Efflux pumps are transport proteins that extrude toxic substances, including antibiotics, from the bacterial cells [1]. They confer resistance to a broad spectrum of antibiotics like macrolides, tetracyclines, and fluoroquinolones [2].

Of the five families of efflux pumps, RND efflux pumps are specific to gram-negative bacteria $[3,4]$. In E. coli and $K$. pneumoniae they are represented by the tripartite AcrAB-TolC system. AcrB is a transporter protein on the inner cell membrane that captures substrates in the cytoplasm or inner membrane of the cell envelope. The protein then transfers it to the outer membrane protein channel TolC, which then transfers it to the external medium. AcrA is a periplasmic accessory protein that mediates the cooperation between AcrB and TolC [5, 6].

Due to the fact that AcrB plays a key role in binding to antibiotics, it is often the target for potential efflux pump inhibitors. Numerous crystal structures of the homotrimer AcrB are available in the Protein Data Bank (PDB), which indicate the binding site of antibiotics and other substrates, as well as identified EPIs.

Punigratane, a novel pyrrolidine alkaloid isolated from the rind of Punica granatum, has been shown to be a potential efflux pump inhibitor [7]. While Punigratane was found to have EPI activity, its mechanism of action is unknown.
In this study, we decided to simulate by computational docking analysis and determine whether the potential EPI Punigratane has the ability to interact with RND efflux pump AcrB and inhibit it. In silico analysis of Punigratane was also carried out to determine the viability of the compound as a drug.

\section{MATERIALS AND METHODS}

\section{Docking analyses}

AcrB target proteins were obtained from the Protein Data Bank (PDB) (http://www.rcsb.org/pdb/home/home.do). The compound Punigratane PDB file was obtained by using the Online SMILES Translator and Structure File Generator (https://cactus. nci.nih.gov/translate/). Representation of Punigratane structure in SMILES: CCCCCCCC1CCC (CCCCCCC) N1C.

Computational docking analyses was carried out using the online platform Mcule, in which docking is carried out by AutoDockVina with default parameters (https://mcule.com/ apps/1-click-docking/) and PatchDock [8]. Docking was carried out based on default parameters with the root mean square (RMS) deviation set at 4.0 tolerance. In the case of AcrB protein 5ENO, docking was based on grid mapping where the cocrcytsal MBX2319 was removed and docking was carried out with Punigratane.

From PatchDock, compounds with the highest score based on geometric shape complementarity were chosen. The area (approximate interface area of the complex) and ACE (Atomic 
contact energy) which is used to calculate desolvation of free energy, were also noted $[8,9]$.

Docking was visualized using PyMol [10], Maestro, and Chimera.

List of AcrB Structures used from PDB database

- 1T9Y (AcrB+PAßN)

- 1T9X $(\mathrm{AcrB}+\mathrm{EtBr})$

- 1T9V (AcrB+Rhodamine 6G)

- 1T9U (AcrB+Ciprofloxacin)

- 2W1B (AcrB+Bile acid)

- 5ENO (AcrB+MBX2319)

- 10YE (AcrB+Ciprofloxacin)

\section{Drug-likeness of a compound}

The drug-likeness of punigratane was predicted using Pre ADMET online tool (https://preadmet.bmdrc.kr/druglikeness/). Based on different rules like CMC like Rule, Lead like Rule, MDDR like Rule, Lipinski's Rule (Rule of Five) and WDI like Rule, the drug-likeness of a compound is predicted. Drug classification was carried out using super pred, which is a prediction web server (http:// prediction.charite.de/) [11].

\section{ADME and toxicity predictions}

Toxicity was tested using toxicity checker at mcule, which is an online drug discovery platform (https://mcule.com/apps/toxicitychecker/). ADME (absorption, distribution, metabolism, and excretion) and overt toxicity properties were simulated using a webbased application admetSAR available at (http://lmmd.ecust. edu.cn: 8000/predict/).

\section{RESULTS AND DISCUSSION \\ Computational docking of punigratane}

Punigratane was computationally docked with 57 AcrB crystal structures available at the PDB database. Each of the AcrB structures had cocrystals (ligands/inhibitors) bound to it, indicating active sites. Docking was performed to check the binding nature of Punigratane in the active site regions, which provides information regarding its inhibitory action.

Bacteria like E. coli and K. pneumoniae which are enteric pathogens, need to tolerate detergents like bile salts which can disrupt their membranes. As the outer membrane is not completely impermeable to bile salts, the AcrAB-TolC efflux pumps also play a major role in bile acid efflux [12]. Studies have shown that deoxycholic acid (bile acid) binds to the AcrB protein in the periplasmic region before being effluxed out of the cell (PDB ID code 2W1B) [13].

When subjected to computational docking analyses, Punigratane was also found to bind in this periplasmic region (table 1). While the cocrystal bile acid was found to interact with S715 and Phe664, Punigratane docked next to it and exhibited hydrophobic interactions with T652, F666, T648, M649, N667, E645, D566, T678, A677 and F563. We hypothesize that Punigratane may act by binding to a region close to the efflux pump substrate, and bring about inhibition by steric hindrances (fig. 1). The EPI PaßN, which is a competitive inhibitor, is also found to bind in a similar manner [14]. This further supports the probability of Punigratane being an EPI.

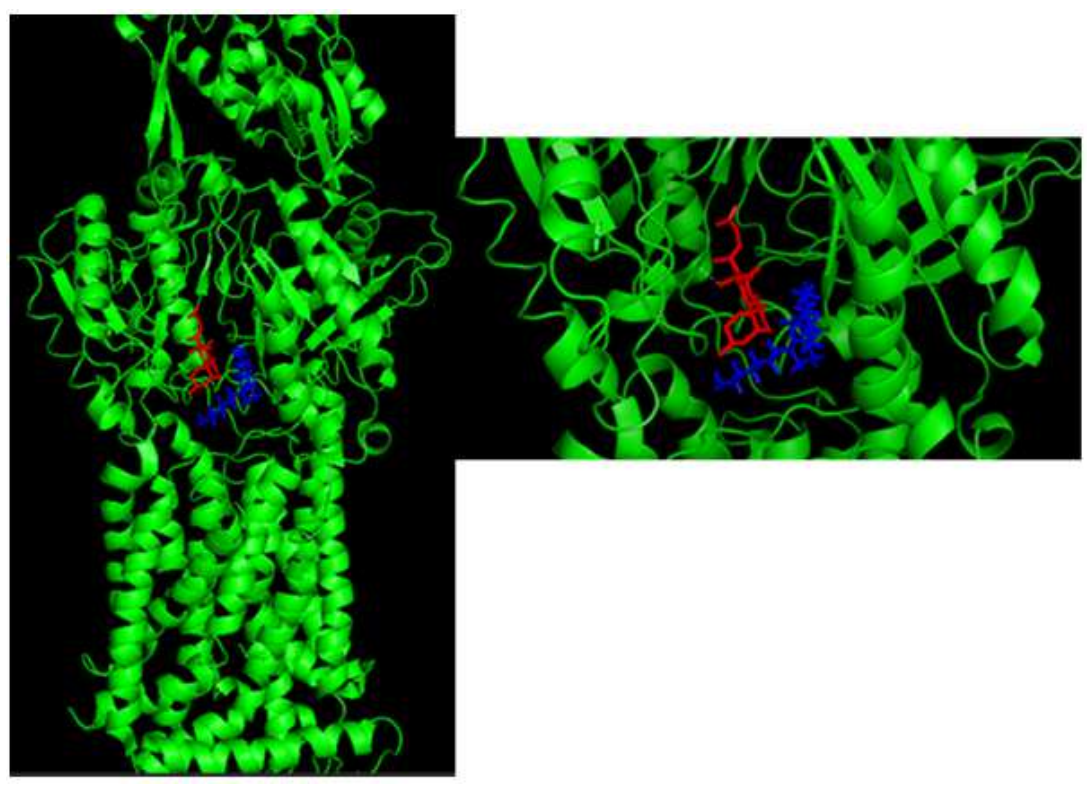

Fig. 1: Docking of Punigratane to AcrB (PDB id: 2W1B). Red and blue indicates Bile Acid (Deoxycholic Acid) and Punigratane molecule, respectively

Punigratane was also found to bind near the ligands of an AcrB mutant. As stated before, AcrB interacts with TolC to expel compounds from within the cell. Previous studies reported that the proteins are connected through a pore, which leads to a large central cavity in AcrB. Drugs like ciprofloxacin, dequalinium, rhodamine 6G, and ethidium bind to upper portion of the central cavity in the AcrB protein, where the pore connecting to TolC is blocked. To allow for this, a mutant AcrB N109A was constructed (Asn109Ala) which exhibited the same effluxing activity as the wild-type [15].

When Punigratane was docked with it, it was found to exhibit similar positions to PaßN (PDB Id: 1T9Y), EtBr (PDB Id: 1T9X), Rhodamine 6G (PDB Id: 1T9V) and Ciprofloxacin (PDB Id: 1T9U) despite their structural differences (table 1). Bile acids were also found to bind in the same region [13].

While this AcrB crystal structure is a hypothetical model, it was interesting to note that both, the substrates $(\mathrm{EtBr}$, Rhodamine 6G, Ciprofloxacin, Bile acid), and inhibitors $(\mathrm{Pa} \beta \mathrm{N})$ bound in the same periplasmic site. Studies suggests that periplasmic binding may occur after the binding of ligands to the central cavity $[13,15]$. The fact that Punigratane was also found in the same region further increases the probability of it being able to bind to AcrB giving further support to its role as an EPI. Fig. 2 represents the surface view of AcrB and depicts the binding pocket where the substrates and the EPI are found. 
Table 1: Docking of punigratane to AcrB structure

\begin{tabular}{llll}
\hline PDB Id (Protein+Cocrystal) & \multicolumn{2}{l}{ Docking of punigratane } & ACE \\
\cline { 2 - 4 } & Docking score & Area & -128.41 \\
\hline 1T9Y (AcrB+PAßN) & 5978 & 676.20 & -165.31 \\
1T9X (AcrB+EtBr) & 5810 & 652.00 & -143.98 \\
1T9V (AcrB+Rhodamine 6G) & 5754 & 651.00 & -148.03 \\
1T9U (AcrB+Ciprofloxacin) & 5832 & 664.70 & -196.21 \\
2W1B (AcrB+Bile acid) & 5634 & 652.20 & \\
\hline
\end{tabular}
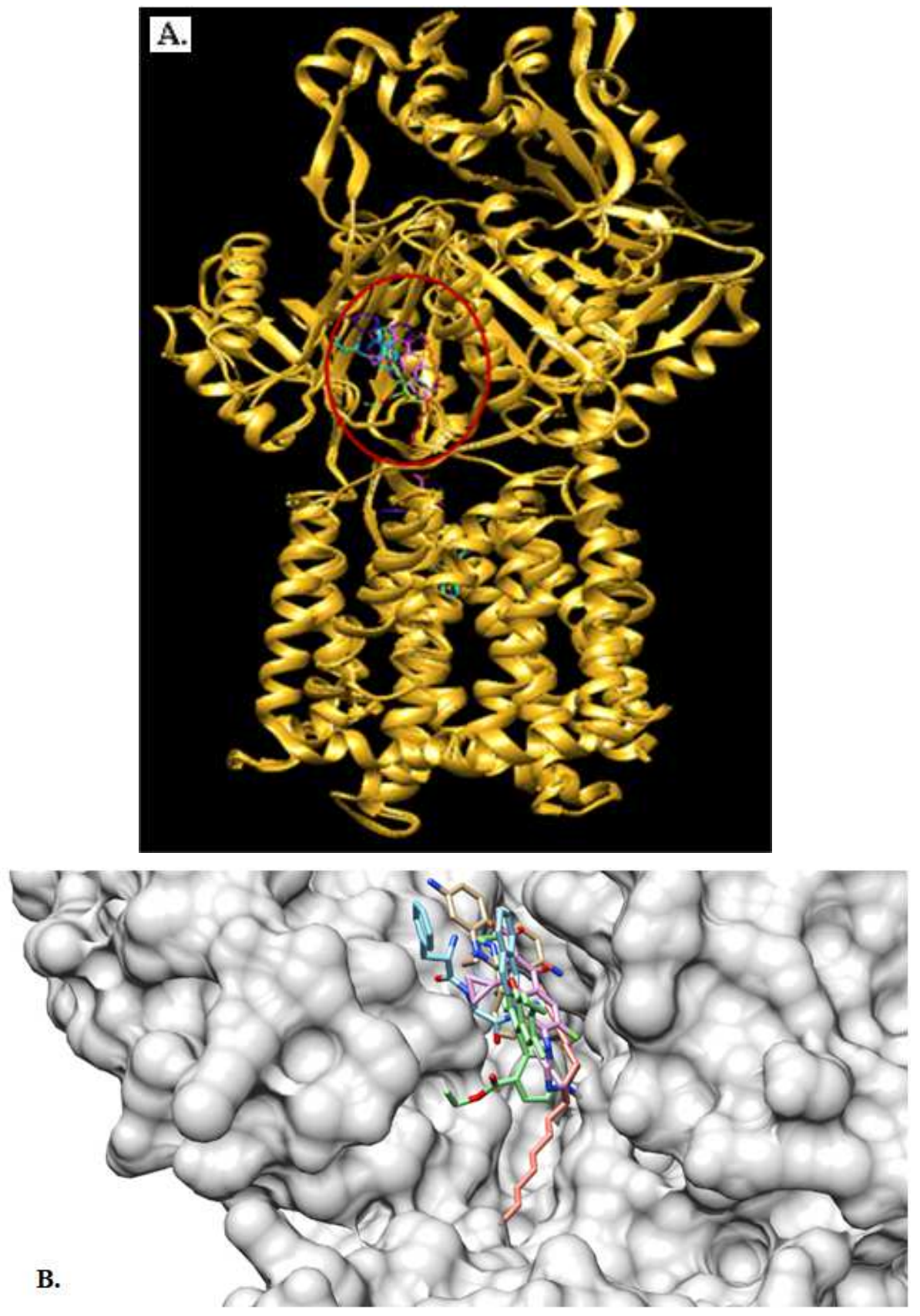

Fig. 2: Docking of punigratane to AcrB mutant. A. Compounds bound to a periplasmic site in AcrB; B. Surface view of AcrB. PaßN, EtBr, Rhodamine 6G, Cipro and Punigratane are represented in in light blue, purple, green, brown and red, respectively

To determine how Punigratane would dock in the active site of an inhibitor, the crystal structure of AcrB with MBX2319 was used (PDB id: 5ENO). MBX2319 is a pyrazolopyridine that binds to AcrB of Escherichia coli and inhibits it [16, 17]. The inhibitor MBX2319 was removed and Punigratane was docked in the same region by uploading the coordinates. It was found to have a docking score of7.8 which is comparable to the cocrystal docking score of-9.3. Punigratane was also found to have the same hydrophobic interactions as MBX2319 (fig. 3). This further supports the probability of Punigratane binding to AcrB and inhibiting it. 

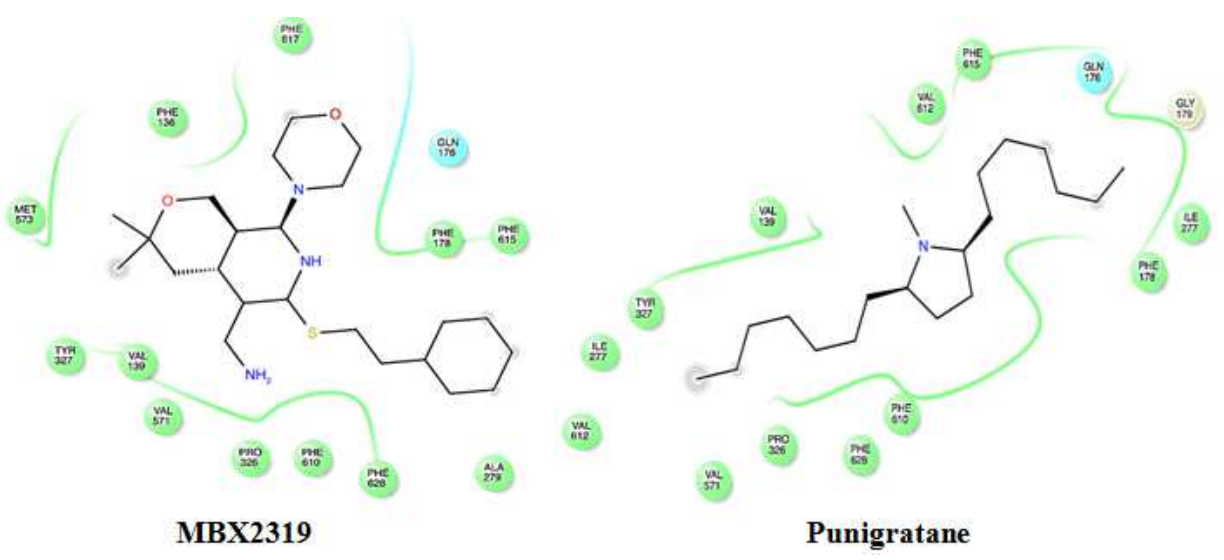

Fig. 3: Hydrophobic interactions of MBX2319 and punigratane with AcrB

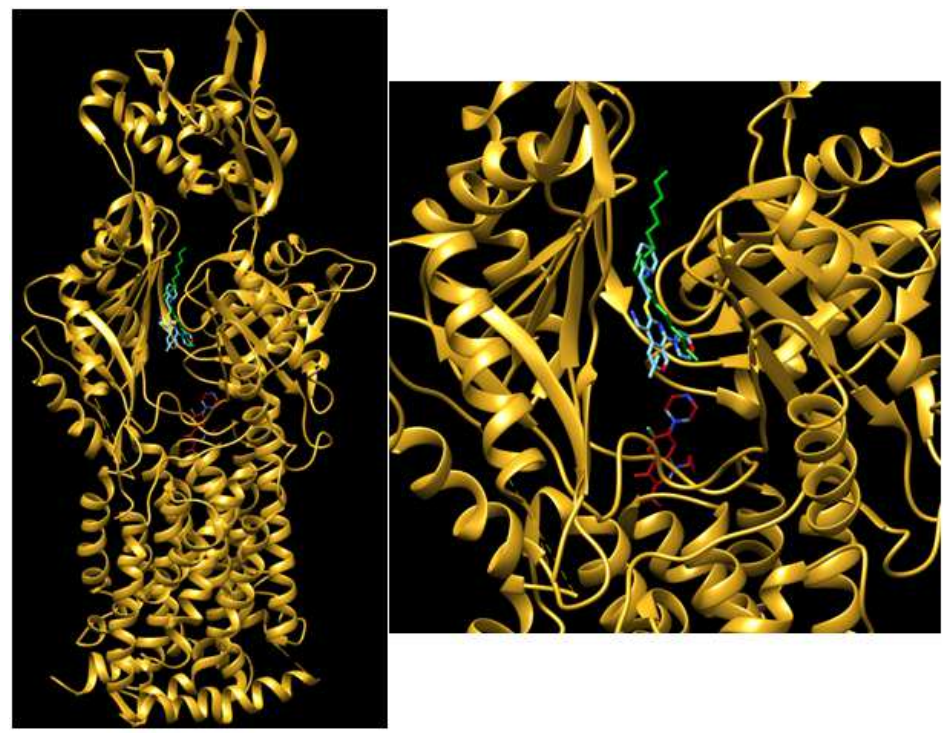

Fig. 4: Docking of punigratane (Green) And MBX2319 (Blue) With AcrB structure+ciprofloxacin (Red) (PDB Id: 10YE)

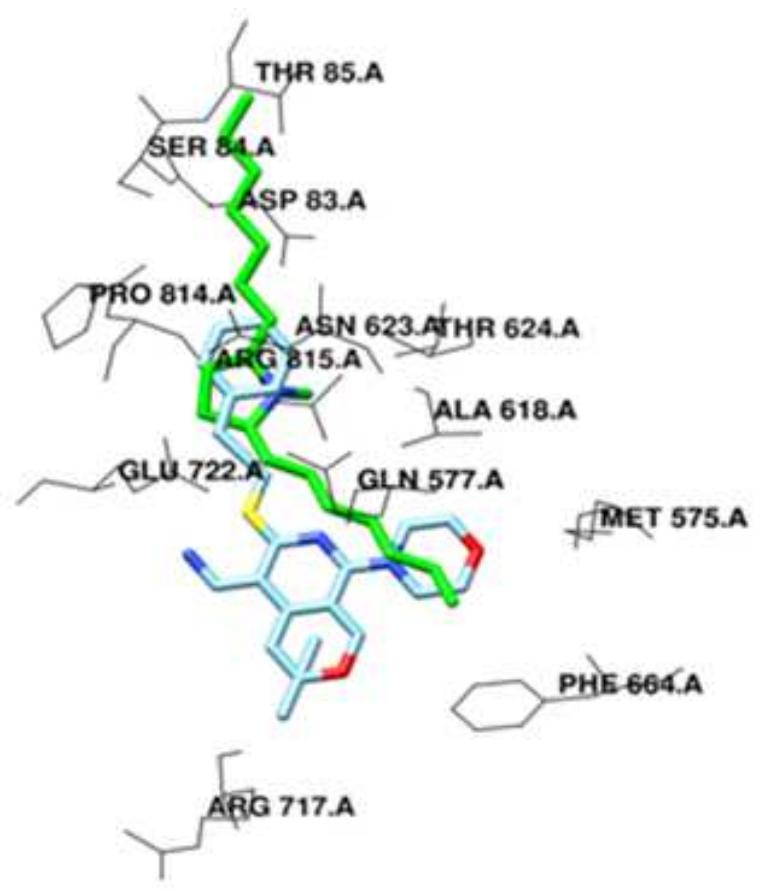

Fig. 5: Hydrophobic interactions of MBX2319 and Punigratane with AcrB (PDB Id: 10YE) 


\section{(PDB id: 5ENO)}

Based on the binding nature of punigratane in the active site of the EPI, Punigratane and MBX2319 were docked with the AcrB structure+Ciprofloxacin (PDB Id: 10YE). Punigratane docking score was 5610, area 666.70, ACE-170.85 and MBX2319 had docking score
5490, area 600.50, ACE-205.81. This gives us a visual representation of where Punigratane and MBX2319 bind with respect to ciprofloxacin (fig. 4). Both Punigratane and MBX2319 bound in the same region with similar hydrophobic interactions (fig. 5). This further supports the probability of Punigratane binding to AcrB and inhibiting it.

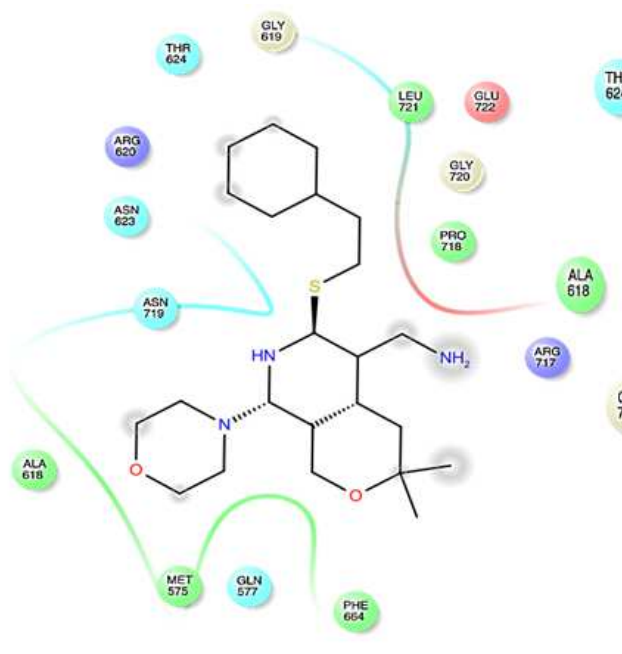

MBX2319

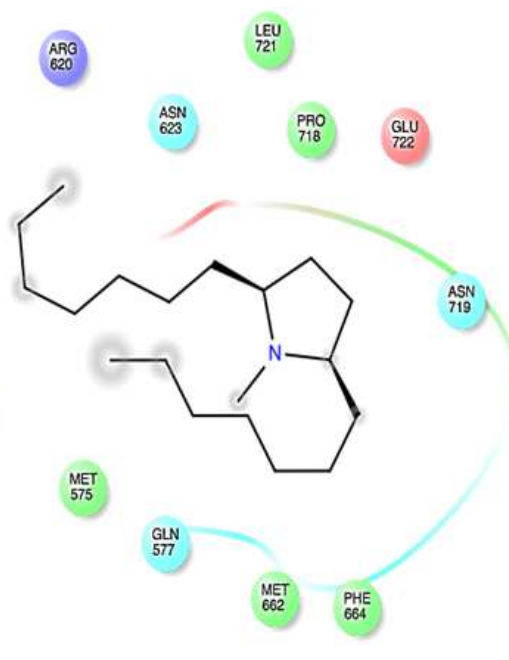

Punigratane

Fig. 5: Hydrophobic interactions of MBX2319 and punigratane with AcrB

\section{(PDB Id: 10YE)}

Therefore based on these observations, we find that Punigratane binds in the periplasmic region of AcrB next to the substrate bile acid. Due to this close location, we hypothesize that it might generate a steric hindrance resulting in blocking of the efflux pump.

The docking score and hydrophobic interactions of Punigratane were comparable to the AcrB inhibitor MBX2319. Hydrophobic interactions play a big role in drug design and are often the driving force of many physiological interactions. All this data suggests that Punigratane would be a potential inhibitor of AcrB. Further refinement and studies including pharmacophore modelling and dynamics will be required to determine the exact binding site and mode of inhibition of Punigratane.

Once the efflux inhibition activity was confirmed by wet lab studies and interaction with efflux pumps by computational docking was studied, other parameters were tested to check the viability of the compound as a drug.

\section{In silico analysis of punigratane}

Punigratane was found to be a potential efflux pump inhibitor by the norfloxacin accumulation assay and was predicted to bind to the AcrB efflux pump by computational docking. To further understand its nature and determine its possible viability as a drug, other parameters were tested.

\section{Drug-likeness}

The drug-likeness of Punigratane was predicted using PreADMET online tool. Based on different rules, the likeness of a compound to be a drug is determined (table 2). The drug-likeness of two inhibitors were also tested as a control. MBX2319 binds to AcrB and inhibits it while $\mathrm{Pa} \beta \mathrm{N}$ binds to AcrB and works on the principle of competitive inhibition. These two inhibitors have different mechanisms of action and bind to different sites on the AcrB protein. Comparing Punigratane to these two compounds could help us predict how it may act. It could also help us determine the viability of Punigratane as a potential drug.

In the PreADMET server, drug-likeness is predicted based on five rules. CMC like Rule indicated that Punigratane and MBX2319 were not a part of the Comprehensive Medicinal Chemistry database while $\mathrm{Pa} \beta \mathrm{N}$ was. All three compounds were found to violate the Lead like Rule indicating that they had a higher probability of being drug-like. MDDR like Rule showed that both Punigratane and MBX2319 were 'mid-structure' while $\mathrm{Pa} \beta \mathrm{N}$ was classified as Drug-like. This means that Punigratane and MBX2319 lie between a drug like and non-drug like compounds based on the MDL Drug Data Report (MDDR) database, which contains compounds with demonstrated biological activities. Lipinski's Rule (Rule of Five) stated that all three compounds were 'suitable' implying that they were likely to be orally active drugs in humans $[18,19]$. WDI like Rule stated that all three compounds were out of $90 \%$ cutoff indicating that it was not a part of the World Drug Index (ChemDB database http://chemdb.kisti.re.kr/help/12_drug. html\#a2). The absence of Punigratane from the different drug databases could be explained due to the novelty of the compound.

Therefore from the data obtained, all three inhibitors had similarities, and Punigratane exhibited higher similarity to MBX2319. It also indicated that Punigratane had drug-like qualities increasing the probability of it being a potential drug.

Table 2: Computational prediction of drug-likeness properties of inhibitors

\begin{tabular}{llll}
\hline Rule & Punigratane & MBX2319 & PaßN \\
\hline CMC like Rule & Not qualified & Not qualified & Qualified \\
Lead like Rule & Violated & Violated & Mid-structure \\
MDDR like Rule & Mid-structure & Suitable & Drug-like \\
Lipinski's Rule (Rule of Five) & Suitable & Out of 90\% cutoff & Suitable \\
WDI like Rule & Out of $90 \%$ cutoff & Out of 90\% cutoff & \\
\hline
\end{tabular}




\section{Comparison to similar drugs}

Punigratane was found to have drug-like characteristics and was also analyzed for the similarities to commercially available drugs. Using super pred, which is a prediction web server, drug classification was carried out. This software uses the Anatomical Therapeutic Chemical (ATC) classification system published by the World Health Organization (WHO) to generate similar drugs to the compound of interest.

It was found that Punigratane was most similar (51.67\%) to Docosanol $\left(\mathrm{C}_{22} \mathrm{H}_{46} \mathrm{O}\right)$, which is an antiviral drug. It showed $25.1 \%$ similarity to the anaesthetic Polidocanol $\left(\mathrm{C}_{14} \mathrm{H}_{30} \mathrm{O}_{2}\right)$ and to the quaternary ammonium compound Cetrimide $\left(\mathrm{C}_{19} \mathrm{H}_{42} \mathrm{BrN}\right)$. Quaternary ammonium compounds have also been shown to have antimicrobial activity and are used to control bacterial growth in clinical and industrial environments (table 3).

By this drug classification, we hypothesize that Punigratane might be classified as an antimicrobial agent. This will further add to its EPI nature.

\section{ADME and toxicity predictions}

Determination of characteristics like Absorption, Distribution, Metabolism, Excretion (ADME) and Toxicity, play a very important role in the viability of a drug. Punigratane was subjected to in silico analysis to predict these properties.

Using Toxicity Checker at Mcule which is an online drug discovery platform (https://mcule. com/apps/toxicity-checker/), it was found that Punigratane had no toxic substructures. ADME (absorption, distribution, metabolism, and excretion) and overt toxicity properties were simulated using admetSAR (table 4) [20]. These criteria are important and play a role in determining the pharmacological activity of a drug. It also helps determine whether a new potential drug is suitable to be taken to the next level of evaluation. Efflux pump inhibitors MBX2319 and PA $\beta N$ were used as controls to compare our data against.

Absorption plays an important role in the bioavailability of a drug. The blood-brain barrier, which is a physiological barrier that restricts passage across it, was permeable to the three inhibitors Punigratane, MBX2319 and PaßN. Punigratane had a higher probability value compared to the other two inhibitors. Human intestinal absorption (HIA) and simulated absorption through Caco2 intestinal cell lines was also estimated. The Caco-2 probability value was highest for Punigratane while the HIA value for MBX2319 was the highest followed by Punigratane then PaßN (table 4). This data indicates that Punigratane would be an effective drug due to its easy absorption.

P-glycoprotein (P-gp) is an $\mathrm{ABC}$ transporter that plays a role in multiple-drug resistance to antitumour agents. A non-substrate would indicate that P-gp would not recognise the molecule and efflux it out [21]. Punigratane was neither a P-gp substrate nor inhibitor while MBX2319 was both and $\mathrm{Pa} \beta \mathrm{N}$ was only a substrate.
Therefore Punigratane was the only compound having no interaction with P-gp, decreasing the probability of it being effluxed out. It was also seen that none of the compounds were substrates for the Renal Organic Cation Transporter indicating reduced expulsion through urine (table 4). These factors increase the probability of Punigratane remaining in the body and acting as a drug.

Cytochrome P450 (CYP450) is a family of enzymes that metabolize xenobiotics. CYP3A4 and CYP2D6 in particular play a role in drug metabolism. CYP substrates indicate compounds that are degraded by the enzymes while inhibitors are compounds that lead to increased drug concentration [22]. Punigratane and PaßN were neither substrates nor inhibitors indicating longevity within the body. They were classified as Low CYP Inhibitory Promiscuity indicating low potency in inhibiting cytochromes [23]. On the other hand, MBX2319 was classified as High CYP Inhibitory Promiscuity and was predicted to be a substrate for CYP450 3A4 and inhibitor of CYP450 2C9, CYP450 2C19 and CYP450 1A2 (table 4). Therefore since Punigratane was neither a substrate nor an inhibitor of cytochromes, it increases its efficacy as a potential drug.

Toxicity is one of the main reasons why bioactive compounds fail to reach the market. In silico tools like these can be used to predict the toxicity of potential drugs [24]. All the three compounds were found to be non inhibitors of the Human Ether-a-go-go-Related Gene (hERG) which codes for potassium (K+) channels that play a role in cardiac action potential repolarization (Zhou et al. 2011). They also tested negative for the Ames test for probable mutagenesis and were non-carcinogenic in nature. The non Ames toxic value was the highest for Punigratane compared to the other two inhibitors. Simulation of LD 50 value (amount needed to cause the death of $50 \%$ of the test animals) resulted in a $2.5 \mathrm{~mol} / \mathrm{kg}$ for all the three inhibitors (table 5). The simulated prediction indicated that Punigratane would be non-toxic in nature, thereby increasing its potency as a drug.

Therefore the data suggests that Punigratane could be a potential drug due to its intestinal absorption, increased bioavailability (as it is not a substrate for cytochrome enzymes) and non-toxic nature. As this is just a simulation, further studies are required to confirm its role as a drug. In the case of the inhibitor $\mathrm{Pa} \beta \mathrm{N}$, while in silico studies indicate it would be a potential drug, studies have shown that due to phototoxicity it cannot be used clinically [5].

In the case of Punigratane, as it has been isolated from the fruit Punica granatum that is consumed worldwide, the probability of toxicity is very low. It is a fruit that is often used in Ayurvedic medicine and toxicity studies carried out in animals, show that the concentrations used in traditional medicine have no deleterious effects and are the same when compared to control groups [26].

Further confirmatory studies will be required to confirm the role of Punigratane as a potential drug that inhibits bacterial efflux pump activity.

Table 3: Comparison of punigratane to similar drugs

\begin{tabular}{|c|c|c|c|c|}
\hline Drug & Similarity to punigratane & Type of drug & Structure & \\
\hline Docosanol (C22H46O) & $51.67 \%$ & antiviral & & \\
\hline Polidocanol (C14H3002) & $25.1 \%$ & anaesthetic & & \\
\hline Cetrimide (C19H42BrN) & $25.1 \%$ & $\begin{array}{l}\text { quaternary ammonium } \\
\text { compound }\end{array}$ & & $\begin{array}{c}\mathrm{CH}_{3} \\
{ }_{13} \mathrm{C}-\mathrm{N}^{*}-\mathrm{CH}_{3}\end{array}$ \\
\hline
\end{tabular}


Table 4: In silico absorption, distribution and toxicity profile

\begin{tabular}{|c|c|c|c|c|c|c|}
\hline \multirow[t]{2}{*}{ Ligand model } & \multicolumn{2}{|c|}{ Punigratane } & \multicolumn{2}{|c|}{ MBX2319 } & \multicolumn{2}{|l|}{ PaßN } \\
\hline & Result & Probability & Result & Probability & Result & Probability \\
\hline Blood-Brain Barrier & + & 0.99 & + & 0.9308 & + & 0.9599 \\
\hline Human Intestinal Absorption & + & 0.9859 & + & 0.9915 & + & 0.9287 \\
\hline Caco-2 Permeability & + & 0.7195 & + & 0.518 & - & 0.6183 \\
\hline P-glycoprotein Substrate & - & 0.6769 & + & 0.5851 & + & 0.7192 \\
\hline P-glycoprotein Inhibitor & - & 0.5795 & + & 0.8483 & - & 0.8048 \\
\hline Renal Organic Cation Transporter & - & 0.5872 & - & 0.5477 & - & 0.6303 \\
\hline CYP450 2C9 Substrate & - & 0.8443 & - & 0.7705 & - & 0.804 \\
\hline CYP450 2C9 Inhibitor & - & 0.828 & + & 0.8949 & - & 0.7968 \\
\hline CYP450 2C19 Inhibitor & - & 0.7331 & + & 0.8994 & - & 0.6298 \\
\hline CYP450 2D6 Substrate & - & 0.6444 & - & 0.6789 & - & 0.6795 \\
\hline CYP450 2D6 Inhibitor & - & 0.8495 & - & 0.9231 & - & 0.7204 \\
\hline CYP450 3A4 Substrate & - & 0.5521 & + & 0.6032 & - & 0.6113 \\
\hline CYP450 3A4 Inhibitor & - & 0.9751 & - & 0.6186 & - & 0.5546 \\
\hline CYP450 1A2 Inhibitor & - & 0.6984 & + & 0.9107 & - & 0.5333 \\
\hline CYP Inhibitory Promiscuity & Low & 0.7984 & High & 0.7797 & Low & 0.7785 \\
\hline CYP450 2C9 Substrate & - & 0.8443 & - & 0.7705 & - & 0.804 \\
\hline
\end{tabular}

+indicates positive ability,-indicates negative ability.

Table 5: In silico etmabolism profile

\begin{tabular}{|c|c|c|c|c|c|c|}
\hline \multirow[t]{2}{*}{ Ligand model } & \multicolumn{2}{|l|}{ Punigratane } & \multicolumn{2}{|l|}{ MBX2319 } & \multicolumn{2}{|l|}{ PaßN } \\
\hline & Result & Probability & Result & Probability & Result & Probability \\
\hline $\begin{array}{l}\text { Human Ether-a-go-go-Related } \\
\text { Gene Inhibition }\end{array}$ & Non-inhibitor & 0.7942 & Weak inhibitor & 0.8768 & Weak inhibitor & 0.9341 \\
\hline AMES Toxicity & Non AMES toxic & 0.8483 & Non AMES toxic & 0.6167 & Non AMES toxic & 0.5537 \\
\hline Carcinogens & Non-carcinogens & 0.8557 & Non-carcinogens & 0.8506 & Non-carcinogens & 0.8908 \\
\hline Biodegradation & $\begin{array}{l}\text { Not ready } \\
\text { biodegradable }\end{array}$ & 0.6482 & $\begin{array}{l}\text { Not ready } \\
\text { biodegradable }\end{array}$ & 1 & $\begin{array}{l}\text { Not ready } \\
\text { biodegradable }\end{array}$ & 0.9566 \\
\hline Model & Value & Unit & Value & Unit & Value & Unit \\
\hline Rat Acute Toxicity & 2.5814 & $\begin{array}{l}\mathrm{LD} 50, \\
\mathrm{~mol} / \mathrm{kg}\end{array}$ & 2.5869 & $\begin{array}{l}\mathrm{LD} 50, \\
\mathrm{~mol} / \mathrm{kg}\end{array}$ & 2.5814 & $\begin{array}{l}\mathrm{LD} 50, \\
\mathrm{~mol} / \mathrm{kg}\end{array}$ \\
\hline
\end{tabular}

\section{CONCLUSION}

Punigratane was found to have potential efflux pump inhibitory activity and from computational docking studies, we hypothesize that it might bind to the AcrB efflux pump. It was found to dock in the periplasmic region of AcrB next to the substrate bile acid (deoxycholic acid) and we predict that it may bring about inhibition due to steric hindrances. It was also found to have a similar docking score and hydrophobic interactions as the AcrB inhibitor MBX2319 suggesting its role as a potential inhibitor of AcrB. When studied with AcrB mutant (AcrB N109A), it was found to bind in the same periplasmic site as the substrates (EtBr, Rhodamine 6G, Ciprofloxacin, Bile acid) as well as the inhibitor $(\mathrm{Pa} \beta \mathrm{N})$ further supporting its ability to bind to AcrB and inhibit it.

Its viability as a drug was also simulated. In silico analysis suggested that Punigratane could be a viable drug due to its intestinal absorption, increased bioavailability and non-toxic nature.

All this data suggests that Punigratane could be a potential drug candidate that has efflux pump inhibition activity and works by binding to the AcrB efflux pump and inhibiting it.

\section{AUTHORS CONTRIBUTIONS}

The first author carried out most of the experimental part of the work. The second author contributed to a part of the study. The third author guided and monitored the experimental design, data compilation, and corrected the manuscript.

\section{CONFLICT OF INTERESTS}

The authors do not have any conflict of interest to declare.

\section{REFERENCES}

1. Webber MA, Piddock LJV. The importance of efflux pumps in bacterial antibiotic resistance. J Antimicrob Chemother 2003;51:9-11.
2. Alekshun MN, Levy SB. Molecular mechanisms of antibacterial multidrug resistance. Cell 2007;128:1037-50.

3. Anes J, McCusker MP, Fanning S, Martins M. The ins and outs of RND efflux pumps in Escherichia coli. Front Microbiol 2015;6:587.

4. Suresh M, Nithya N, Jayasree P, Manish Kumar P. Detection and prevalence of efflux pump-mediated drug resistance in clinical isolates of multidrug-resistant gram-negative bacteria from North Kerala, India. Asian J Pharm Clin Res 2016;9:324-7.

5. Piddock LJV. Clinically relevant chromosomally encoded multidrug resistance efflux pumps in bacteria clinically relevant chromosomally encoded multidrug resistance efflux pumps in bacteria. Clin Infect Dis 2006;19:382-402.

6. Gupta P, Rai N, Gautam P. Anticancer drugs as potential inhibitors of AcrAB-TolC of multidrug-resistant E. coli: an in silico molecular modelling and docking study. Asian J Pharm Clin Res 2015;8:351-8.

7. Rafiq Z, Narasimhan S, Vennila R, Vaidyanathan R. Punigratane, a novel pyrrolidine alkaloid from punica granatum rind with putative efflux inhibition activity. Nat Prod Res 2016;6419:1-6.

8. Schneidman Duhovny D, Inbar Y, Nussinov R, Wolfson HJ. PatchDock and SymmDock: servers for rigid and symmetric docking. Nucleic Acids Res 2005;33:W363-7.

9. Mashiach E, Schneidman Duhovny D, Peri A, Shavit Y, Nussinov $\mathrm{R}$, Wolfson HJ. An Integrated suite of fast docking algorithms. Proteins: Struct Funct Bioinf 2010;78:3197-204.

10. Schrodinger LLC. The PyMOL molecular graphics system, Version 1.8; 2015.

11. Nickel J, Gohlke BO, Erehman J, Banerjee P, Rong WW, Goede A, et al. SuperPred: update on drug classification and target prediction. Nucleic Acids Res 2014;42:W26-31.

12. Thanassi DG, Cheng LW, Nikaido H. Active efflux of bile salts by Escherichia coli. J Bacteriol 1997;179:2512-8.

13. Drew D, Klepsch MM, Newstead S, Flaig R, De Gier JW, Iwata S, et al. The structure of the efflux pump AcrB in complex with bile acid. Mol Membr Biol 2008;25:677-82. 
14. Zechini B, Versace I. Inhibitors of multidrug-resistant efflux systems in bacteria. Recent Pat Antiinfect Drug Discovary 2009;4:37-50.

15. Yu EW, Aires JR, McDermott G, Nikaido H. A periplasmic drugbinding site of the AcrB multidrug efflux pump: a crystallographic and site-directed mutagenesis study. J Bacteriol 2005;187:6804-15.

16. Opperman TJ, Kwasny SM, Kim HS, Nguyen ST, Houseweart C, D'Souza S, et al. Characterization of a novel pyrazolopyridine inhibitor of the AcrAB efflux pump of Escherichia coli. Antimicrob Agents Chemother 2014;58:722-33.

17. Nguyen ST, Kwasny SM, Ding X, Cardinale SC, McCarthy CT, Kim $\mathrm{HS}$, et al. Structure-activity relationships of a novel pyrazolopyridine series of Gram-negative bacterial efflux pump inhibitors. Bioorg Med Chem 2015;23:2024-34.

18. Schneider G. Prediction of drug-like properties. Madame Curie Biosci. Database Landes Bioscience; 2013.

19. Pradeep PS, Shrungesh KTO, Prashantha N, Mahadevan KM. Synthesis, in vitro antibacterial, toxicity and molecular docking anticancer activity of novel n-[(2-chloroquinolin-3-yl) methylidene]-2-aniline schiff bases. Int J Curr Pharm Res Acad 2015;7:37-46.
20. Cheng F, Li W, Zhou Y, Shen J, Wu Z, Liu G, et al. admetSAR: a comprehensive source and free tool for assessment of chemical ADMET properties. J Chem Inf Model 2012;52:3099-105.

21. Nisha CM, Kumar A, Vimal A, Bai BM, Pal D, Kumar A. Docking and ADMET prediction of few GSK-3 inhibitors divulges 6bromoindirubin-3-oxime as a potential inhibitor. J Mol Graph Model 2016;65:100-7.

22. Ahmed NH, Raghuraman K, Baruah FK, Grover RK. Antibiotic resistance pattern of uropathogens: an experience from North Indian Cancer patient. J Glob Infect Dis 2015;7:113.

23. Nath A, Zientek MA, Burke BJ, Jiang Y, Atkins WM. Quantifying and predicting the promiscuity and isoform specificity of smallmolecule cytochrome P450 inhibitors. Drug Metab Dispos 2010;38:2195-203.

24. Waterbeemd $\mathrm{H}$ van de, Gifford E. ADMET in silico modelling: towards prediction paradise? Nat Rev Drug Discovery 2003;2:192-204.

25. Zhou P, Babcock J, Liu L, Li M, Gao Z. Activation of human ethera-go-go-related gene (hERG) potassium channels by small molecules. Acta Pharmacol Sin 2011;32:781-8.

26. Jurenka JS. Therapeutic applications of pomegranate (Punica granatum L.): a review. Altern Med Rev 2008;13:128-44. 